\title{
BMJ Open Protocol for a mixed-method cohort study investigating the prevalence and impact of obsessive-compulsive disorder (OCD) in chronic pain rehabilitation
}

\author{
Chad Sloley (D) , ${ }^{1}$ Edward A Shipton, ${ }^{1}$ Caroline Bell, ${ }^{2}$ Jonathan Williman ${ }^{3}$
}

To cite: Sloley C, Shipton EA, Bell C, et al. Protocol for a mixed-method cohort study investigating the prevalence and impact of obsessive-compulsive disorder (OCD) in chronic pain rehabilitation. BMJ Open 2021;11:e052288. doi:10.1136/ bmjopen-2021-052288

- Prepublication history for this paper is available online. To view these files, please visit the journal online (http://dx.doi. org/10.1136/bmjopen-2021052288).

Received 11 April 2021 Accepted 21 July 2021

Check for updates

(c) Author(s) (or their employer(s)) 2021. Re-use permitted under CC BY-NC. No commercial re-use. See rights and permissions. Published by BMJ.

${ }^{1}$ Department of Anaesthesia, University of Otago, Christchurch, New Zealand ${ }^{2}$ Department of Psychological Medicine, University of Otago, Christchurch, New Zealand ${ }^{3}$ Department of Population Health, University of Otago, Christchurch, New Zealand

Correspondence to Chad Sloley; chad.sloley@postgrad.otago. ac.nz

\section{ABSTRACT}

Introduction While there is considerable and growing research in the individual fields of obsessive-compulsive disorder (OCD) and chronic pain, focused research into their potential association remains limited. By exploring this potential association, better theoretical understanding of and better therapeutic approaches to chronic pain management could be developed. The study's aim is to explore the prevalence and impact of obsessionscompulsions on the experience and rehabilitation of chronic pain among individuals attending different branches of a New Zealand pain service.

Methods and analysis This is a cohort study using wellvalidated questionnaires and semistructured interviews. Participants will be recruited through community pain services from a private rehabilitation-focused company with branches across New Zealand. Participants will complete an OCD screening measure (Obsessive-Compulsive Inventory-Revised (OCl-R)). These results will be used to compare results from the specialist pain services benchmarking electronic Persistent Pain Outcomes Collaboration measure sets, at both participant intake and completion of each Pain Service Programme. Prevalence rates of $\mathrm{OCD}$ caseness from the $\mathrm{OCl}-\mathrm{R}$ will be estimated with $95 \% \mathrm{Cl}$. Generalised linear regression models will be used to explore differences in pain baseline and outcome factors between those with high and low obsessivecompulsive symptoms. Semistructured interviews, assessed through interpretative phenomenological analysis (IPA), will be used to provide information on lived experiences of individuals with comorbid chronic pain and OCD. This will be supported through the administration of an Obsessive Beliefs Questionnaire 44.

Ethics and dissemination Ethical approval has been obtained from the Health and Disability Ethics Committee (HDEC20/CEN/82). Study results will be disseminated at professional conferences and in peer-reviewed journals. A lay summary of findings will be provided to requesting participants or through attendance at a local hui (gathering). Trial registration number Australian New Zealand Clinical Trials Registry (ACTRN12621000758808).

\section{INTRODUCTION}

Chronic or persistent, non-cancer pain refers to a heterogeneous group of clinical conditions, in which pain persists or recurs for longer than 3 months. ${ }^{1}$ It represents
Strengths and limitations of this study

- This is the first study to directly explore prevalence, impact and experience of obsessive-compulsive symptoms on chronic pain rehabilitation.

- By using a mixed-method design, the qualitative component will provide rich information, whereas the quantitative component will help provide generalisable estimates of parameters of interest.

- Use of an obsessive-compulsive disorder screening measure limits the burden on potential participants, already dealing with the demands of involvement in pain services, and it encourages greater participation. However, the nature of the information collected via this method is limited as compared with the use of a clinical interview.

- Resource and practical constraints have led to the exclusion of tertiary-level pain services, which limits the inclusion of a certain subset of chronic pain sufferers attending a pain service.

- Response bias considerations associated with a cohort study design.

an important consideration in the New Zealand public health system. A recent national survey $(2017 / 2018$ New Zealand Health Survey) reported that $19.7 \%$ of the population, or an estimated 770000 adults, suffers from chronic pain. ${ }^{2}$ While historical views of chronic pain have been predominantly biomedical in focus, it is increasingly recognised that complex interplays between biological, psychological, sociocultural and economic factors underlie the development and maintenance of chronic pain. ${ }^{3}$ This emphasises the importance of reviewing potential contributing factors as a way of both furthering conceptual understandings and supporting effective clinical interventions. Within this framework, a growing body of research is exploring the role of psychopathology in the transition of acute pain into acute persistent pain and subsequently into chronic (or persistent) pain states as well as 
its role in acting as a significant barrier to intervention or recovery. ${ }^{4}$

Obsessive-compulsive disorder (OCD) is a neuropsychiatric condition, which is heterogeneous and often chronic that affects approximately $1 \%-3 \%$ of the general population. ${ }^{5-7}$ It is a cross-cultural and crosssocioeconomic phenomenon. ${ }^{8}$ Geographical and cultural factors contribute to variability in symptom presentation and frequency. ${ }^{9}$ At its core, it features persistent obsessions and/or compulsions. ${ }^{1011}$ Obsessions are defined as 'recurrent and persistent thoughts, urges or images that are experienced as intrusive and unwanted'. Compulsions are defined as 'repetitive behaviours or mental acts that an individual feels driven to perform in response to an obsession, or according to rules that must be applied rigidly'. ${ }^{10}$

While there is considerable and growing research in OCD, there is limited investigation into its association with chronic pain. While included as part of larger studies on psychopathology comorbidity, to the best of the researchers' knowledge, there has only been one direct study into the prevalence of OCD in chronic pain. This reported a high lifetime prevalence of OCD in a sample of those suffering from chronic pain. ${ }^{12}$ In addition, there has been no direct investigation into the potential impact that OCD has on chronic pain and its rehabilitative processes or outcomes.

However, a review of literature pertaining to OCD indicates the presence of various aspects that have possible important implications for chronic pain sufferers. In particular, OCD is associated with high rates of diagnostic comorbidity. ${ }^{13}$ It overlaps with illness anxiety ${ }^{14}$ and is linked to significant disability and difficulty, ${ }^{6}$ including increased suicidal risk. ${ }^{15}$ Its symptoms are associated with poorer self-reported physical health status ${ }^{16}$ and reduced quality of life. ${ }^{17}$ It is associated with a tendency towards threat overestimation and heightened appraisal of potential negative outcomes. ${ }^{18}{ }^{19}$ It holds a significant association with cognitive rumination, with the latter noted to contribute to pain catastrophisation as well. ${ }^{20}{ }^{21}$ It is associated with impaired functioning of certain neurobiological pathways, including various cortical and subcortical structures, that are linked with complex processes, such as evaluation, affect regulation, rewardbased decision making and goal-directed behaviour. ${ }^{22} 23$ Dysfunction of dorsal-striatal-centric circuitry is seen to contribute to compulsive behaviour but is also implicated in learning habits and in addiction. ${ }^{24}$ These have some possible important repercussions to consider with regard to chronic pain where dopamine and reward/aversion systems are understood to be involved in the pain/analgesia processes and where the presence of pain/analgesia can alter levels of activity of the reward system. ${ }^{25}$ In turn, dopamine is also implicated in striatal functioning ${ }^{24}$ and is conveyed as an important modulator in habit leaning. ${ }^{26}$ OCD is also associated with deficits in organisational skills that lead to impairment across learning strategy use and memory recall, ${ }^{27-29}$ which may have important implications for how individuals suffering from chronic pain engage with rehabilitation-directed information and strategies. Research also points to subjective doubt being an important feature of $\mathrm{OCD},{ }^{30}$ with a nascent $\mathrm{OCD}$ model postulating an attenuated access to internal states (such as emotions, bodily sensations, muscle tension and proprioception). ${ }^{31-33}$

Chronic pain and OCD are complex conditions linked with significant disability and distress. In consideration of the aspects and processes highlighted above, further study into the association and impact of OCD on chronic pain and its rehabilitation is merited.

\section{OBJECTIVES}

The overall aim of this study is to explore the prevalence and impact of obsessive-compulsive symptoms among chronic pain sufferers attending pain services in New Zealand. We hypothesise that OCD displays a significantly higher prevalence rate among chronic pain sufferers, than the general population. We also hypothesise that it is associated with greater complexity and intensity of pain experiences, greater life interference, greater requirement for clinical input through Pain Service Programmes and worse programme outcomes.

To test these hypotheses, this study will:

1. Determine the prevalence of participants with OCD caseness from the Obsessive-Compulsive InventoryRevised (OCI-R) attending an active Pain Service Programme and contrast this with the rates of general population estimates for OCD as derived from previous literature.

2. Determine the degree to which obsessive-compulsive symptoms are associated with pain complexity, pain intensity and daily life interference of individuals that leads into a Pain Service Programme.

3. Determine the degree to which obsessive-compulsive symptoms are associated with greater need for clinical input and pain outcomes through Pain Service Programmes.

4. Explore how individuals make sense of their experiences of co-occurring chronic pain and OCD and how their accounts of obsessions and compulsions contribute to their pain rehabilitation experiences.

\section{METHODS AND ANALYSIS \\ Study design}

This is a cohort study using questionnaires and semistructured interviews with participants recruited through community pain services from a rehabilitation-focused company, Habit Health. Habit Health is one of New Zealand's largest private integrated health, fitness and physiotherapy rehabilitation provider. It incorporates an established community pain service and comprising seven distinct units across New Zealand. New Zealand holds a relatively unique health system approach, where a government entity, the Accident Compensation 
Corporation (ACC), acts as the country's sole accident insurance for all work-related and non-work-related injuries for its populace. As part of its mandate, ACC partners with registered health professionals and private rehabilitation companies, such as Habit Health, in order to provide rehabilitative services. Within this framework, the Habit Health Community Pain Service caters to both private and non-private patient referrals.

Inclusion criteria for the study include all individuals over the age of 18 years involved in an active Community Pain Service Programme and who have sufficient Englishlanguage proficiency to independently complete report measures.

Pain services in New Zealand are specialist multidisciplinary services with a core team comprising a physician, physiotherapist, psychologist and occupational therapist. At a community level, Pain Service Programmes consist of two stages, with individuals with chronic pain issues referred into the first stage. The second stage expands and builds on services delivered in the first stage and includes mandatory medical practitioner input and medication review. Progression into a second stage depends on individual needs, complexity of barriers to pain rehabilitation and resource requirements. As part of standard service practices, electronic Persistent Pain Outcomes Collaboration (ePPOC) data sets are administered at intake and on completion (either at stage one or two depending on the individual's service progression) points of individual Pain Service Programmes.

Recruitment will occur over a period of 22 months, spanning from June 2020 to April 2022. A study flyer, including the primary researcher's (CS) contact details, will be included with initial service documents sent to individuals starting at a pain service (figure 1). Pain service key workers (clinicians performing the initial assessment and managing the overall programme) will also approach already enrolled, and eligible, service clients to inform them of the study and to attain verbal consent for their contact details to be passed on to the primary researcher. The primary researcher would then contact, or be contacted by, assenting individuals to discuss the study details and confirm eligibility. Eligible clients will be emailed a link via REDCap web-based survey to complete informed consent and baseline measures including the OCI-R. Individuals unable to access the online survey will be provided with a physical study pack comprising the study information sheet, the informed consent form, an OCI-R measure and a postage-paid return envelope. The primary researcher will follow up on surveys where there has been no response within 1-2 weeks of the survey initially being sent. This will occur via either email or telephone call.

A consented participant's ePPOC data will be collected at intake and completion points for their pain service as well.

Through purposeful sampling, a small number of individuals indicating OCD-chronic pain comorbidity (either by prior clinical diagnosis or as supported by high OCI-R

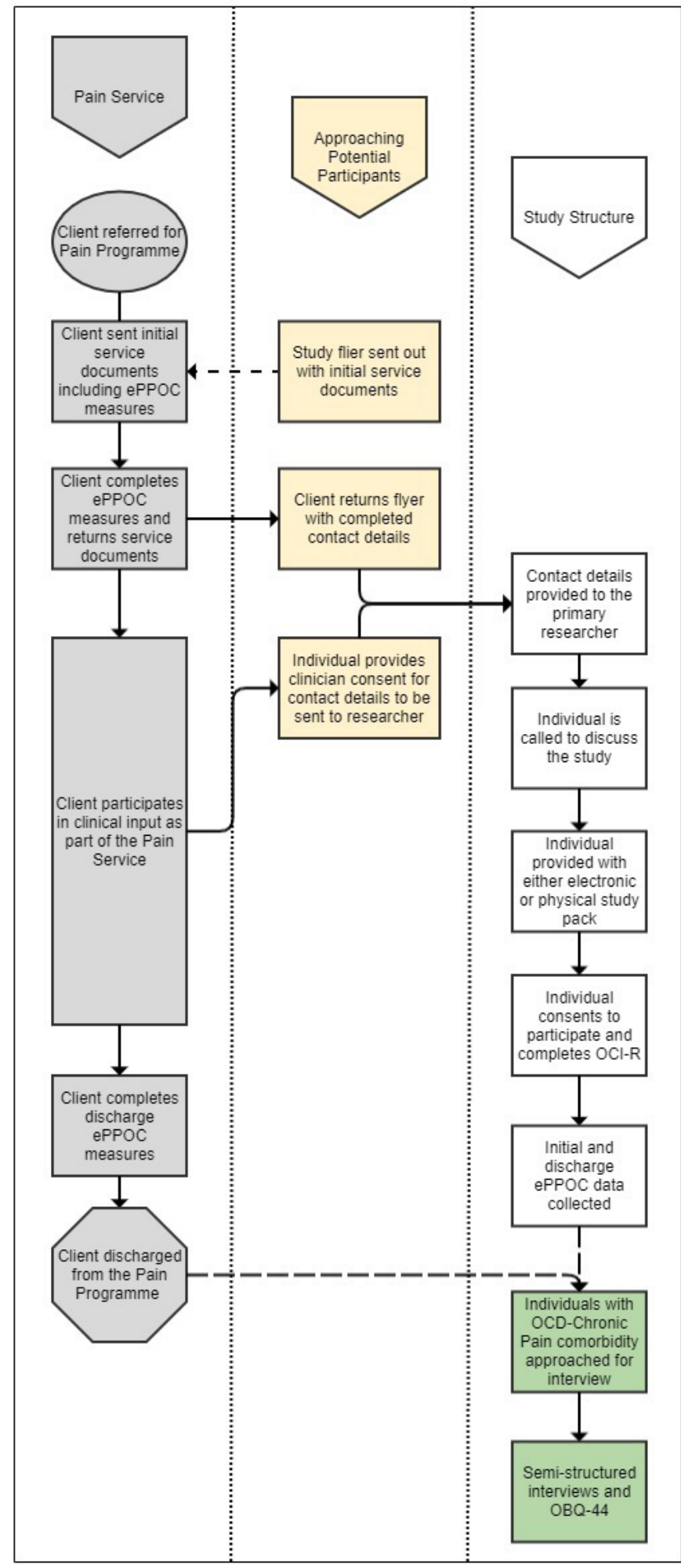

Figure 1 Study structure. ePPOC, electronic Persistent Pain Outcomes Collaboration; OBQ-44, Obsessive Beliefs Questionnaire 44; OCD, obsessive-compulsive disorder; OCl-R, Obsessive-Compulsive Inventory-Revised.

and ePPOC results) will be approached to participate in a semistructured interview. This interview will be conducted following the completion of any active community pain service and will be focused on exploring the experiences 
of their conditions and pain rehabilitation. Interviewees will also complete an obsession-directed self-report measure (Obsessive Beliefs Questionnaire 44 (OBQ-44)) to assist in providing clinical understanding of participant experiences regarding obsessions.

\section{Measures}

Obsessive-Compulsive Inventory-Revised (OCl-R)

The OCI-R is an 18-item self-report measure assessing characteristic symptoms of OCD for their prevalence over the past month. ${ }^{34}$ Items are rated on a 5-point Likert Scale, ranging from 0 (not at all) to 4 (extremely), with higher scores indicating a greater presence of OCDassociated symptoms. A total score of 21 is suggested as an optimal cut-off score for distinguishing the presence of OCD ${ }^{34}$ which will be used by this study to indicate 'caseness' in the absence of a clinician-administered diagnostic assessment. This self-report measure holds good internal consistency, good to adequate short-term testretest reliability and fair convergence with clinician-rated measures of OCD. ${ }^{35}$ Its use as a screening and research tool has been validated in both clinical ${ }^{34}$ and non-clinical samples $^{36}$ as well as within the New Zealand context. ${ }^{37}$

\section{Obsessive Beliefs Questionnaire 44 (OBQ-44)}

The OBQ-44 is a 44 -item questionnaire, assessing across three factors hypothesised to be associated with obsessive-compulsive symptoms and worry. They include (1) responsibility and threat estimation, (2) perfectionism and intolerance for uncertainty and (3) importance and control of thoughts. ${ }^{38}$ This questionnaire displays good internal consistency with a sample of both clinical and non-clinical participants ${ }^{38}$ and correlates with obsessivecompulsive symptoms. ${ }^{39}$

\section{Electronic Persistent Pain Outcome Collaboration (ePPOC)}

ePPOC incorporates a broad range of patient-reported data items and assessment tools to measure various outcomes across physical, functional and psychological aspects from specialist pain services In Australia and New Zealand. ${ }^{40}$ These are completed at intake, completion and 3-6 months postcompletion of an individual pain service by service facilitators. Information is electronically stored. Information contributes to locally held and internationally held rehabilitation service outcome databases. The information and measures, forming part of the ePPOC set, that will be accessed for the purposes of this study include the following.

\section{Patient characteristics}

Date of birth, gender, ethnicity and comorbidities.

\section{Medication use}

Number of major drug groups.

\section{Brief Pain Inventory (BPI)}

Measure assessing the location of pain, its severity and its interference in daily activities.
Pain Self-Efficacy Questionnaire (PSEQ)

A client-reported measure assessing the confidence an individual has in order to perform a range of activities, despite the presence of pain. ${ }^{42}$ Ten items are rated on a scale from 0 to 6 , where $0=$ 'not at all confident' and $6=$ 'completely confident'.

\section{Pain Catastrophizing Scale (PCS)}

A client-reported measure assessing the presence of painrelated thoughts and cognitions that may contribute to more intense pain, increased disability and emotional distress. ${ }^{43}$ Thirteen items are rated on a scale from 0 to 4 , where $0=$ 'not at all' and $4=$ 'all the time'. It provides an overall score as well as subscale scores associated with rumination, magnification and helplessness.

\section{Depression, Anxiety Stress Scale-21 (DASS-21)}

A client-reported measure of the emotional states of depression, anxiety and stress, appropriate for use in both clinical and research settings. ${ }^{44}$ Twenty-one statements are rated on a 4-point scale as to how much they have applied over the past week. It is indicated as a valid and reliable measure with applicability to the persistent pain population. $^{45}$

\section{Healthcare utilisation}

Pain-related utilisation of general practitioner (GP), specialist, allied health services, presentations to emergency department, admission to hospital and diagnostic tests undertaken in the past 3 months.

\section{Data analysis}

OCI-R scores for each participant will be analysed. To answer the question of prevalence rate, an OCI-R total score of 21 (a categorical variable) will be set as a cutoff mark to dichotomise between OCD caseness presence and non-presence. The OCD caseness prevalence rate of participants will be estimated with 95\% 'Wilson' binomial CIs, and rates will be considered greater than the general population if the lower bound of the 95\% CI exceeds $3 \%$. Three percent was chosen as it denotes the upper limit of OCD prevalence rates identified among general population studies.

Associations between obsessive-compulsive symptoms and experiences of chronic pain will be explored through generalised linear regression models, with individuals' OCI-R total scores (a continuous variable) included as the explanatory independent variable. Outcomes of interest include those measured via the ePPOC at baseline, pain catastrophising (PCS), pain intensity and interference ratings (BPI), pain self-efficacy (PSEQ) and length of time that pain has been present. Reported change in pain and in physical abilities between service entry and discharge will be modelled without adjustment for baseline differences. The proportion of participants who transition into the pain service stage 2 will be determined and compared by OCI-R using log-binomial or logistic regression models. 
Count outcome variables will be analysed through use of Poisson regression model. These will include number of times seeing a GP and number of times consulting health professionals prior to the start of the pain service.

Non-linear associations between OCI-R and outcome variables will be modelled using restricted cubic splines. In addition to univariable models, multivariable models will be run with, and adjustment for, potential confounders. The study identifies various confounding variables including age, gender, ethnicity, depression (DASS-21) and medication use (benzodiazepines and opioids).

All planned analysis will be presented together, and interpretation of analysis will depend on consistency of results across all measures rather than identifying individual measures that do, or do not, meet certain $p$ value thresholds.

\section{Sample size}

The sample size is constrained by resource limitations and the number of patients presenting to community pain services in New Zealand. Based on current projections of enrolment, we estimate we will be able to enrol 150 participants during the study recruitment period. This sample size will allow us to estimate the prevalence of OCD caseness with a $95 \% \mathrm{CI}$ of approximately 10 percentage points or less in width.

There is currently little information as to what the prevalence of OCD caseness might be in this patient population. Assuming it is $8 \%$ or higher, it would provide $80 \%$ power to rule out a prevalence of $3 \%$ or less.

The absolute number of patients presenting with OCD caseness is likely to be too small to include as a binary variables in logistic regression models, but associations between explanatory variables and obsessive-compulsive symptoms will be able to be explored using individuals' OCI-R total scores in linear regression models. ${ }^{46}$

\section{Qualitative analysis}

The qualitative component for this study will be performed from a constructivist point of view using an interpretative phenomenological epistemology. In recognising that both OCD and chronic pain conditions are associated with significant complexity, situational impact and individual/subjective meaning, this study will look to explore how individuals who have these conditions co-occurring make sense of their experiences. It will also look at how subjective accounts of obsessions and compulsions contribute to pain rehabilitation experiences.

Semistructured, one-on-one, interviews will be conducted with participants (purposeful sampling) indicating OCD-chronic pain comorbidity (either by clinical diagnosis or as supported by high OCI-R and ePPOC results), as noted through the questionnaire component of the study. These interviews will be conducted following completion of any active community pain service.

An IPA approach will be employed to guide information collection and analyses. All interviews will be audiorecorded, transcribed verbatim and anonymised. The primary investigator (CS), who is a registered clinical psychologist and experienced with both chronic pain/ rehabilitation and mental health clinical assessment and interventional work, will conduct all the interviews. The primary investigator will also undertake detailed caseby-case analyses of the individual transcripts to identify patterns of meaning/themes and formulate towards narrative accounts. Other members of the research team will review these accounts to help support validity of the analyses. Interviews will look to be conducted with a sample of 3-6 participants. This number was based on (1) the aim of this component of the study being an in-depth, rich exploration of individual meaning and experiences rather than on generalisations; (2) consideration of potential occurrences of suitable participants given estimates of OCD population rates and the study's overall projected sample size; and (3) researcher time and resource availability. This sample size fits appropriately within an IPA approach, which emphasises focus on 'lived experiences' and comprehensively exploring personal perceptions and accounts. ${ }^{47} 48$

Interviewees' will also complete an obsession-directed self-report questionnaire (OBQ-44). Interviewee responses to this questionnaire will be qualitatively interpreted to assist in providing clinical understanding of participant experiences of obsessions.

\section{Patient and public involvement}

No patient or public involvement occurred in the design and planning of the study.

\section{Ethics and dissemination}

This study was granted ethical approval by the New Zealand Health and Disability Ethics Committees (20/ CEN/82). Approval was also attained from a private rehabilitation company, Habit Health, to assist in approaching individuals for participation in the study. Participation in the study does not interfere with typical care that individuals receive through their respective pain services. Participants will be able to opt in to the study and will be able to withdraw at any time. The study will look to protect participant anonymity through the use of number coding methods. All information will be securely held. The primary researcher will look to debrief participants should any emotional reactions elicited from completing the surveys occur. In the unlikely event of any safetyrelated concerns being noted, participants will either be referred back to their treating clinicians for further input/care or alternatively be directed to relevant emergency care facilities/entities. Attempts will be made to support culturally appropriate engagement, particularly in relation to the semistructured interview component of the study. As part of this, procedures and approaches surrounding the interview process (eg, venue, greetings, opening and closing customs) will be developed to be responsive to the cultural needs of the individual.

No monetary, or other, incentives will be offered to individuals for their participation. The only anticipated 
direct costs to participants will relate to travel expenses for those involved in the semistructured interviews.

Results from this study will be disseminated at regional and international conferences and in peer-reviewed journals. In addition, a lay summary of the study findings will be sent to all study participants who wish to access these and/or through attendance at a local hui (gathering).

Contributors CS and EAS were involved in the conception of the study. CS, EAS, $\mathrm{CB}$ and JW contributed to the study design. CS prepared the application for ethical approval with input from EAS, CB and JW. CS wrote all programme components. All authors reviewed and critiqued the manuscript and approved the final version.

Funding The authors have not declared a specific grant for this research from any funding agency in the public, commercial or not-for-profit sectors.

Competing interests All authors have completed the ICMJE uniform disclosure at www.icmje.org/coi_disclosure.pdf and declare no support from any organisation for the submitted work; CS is employed on a full-time basis with Habit Health; there are no other relationships or activities that could appear to have influenced the submitted work.

Patient and public involvement Patients and/or the public were not involved in the design, conduct, reporting or dissemination plans of this research.

Patient consent for publication Not required.

Provenance and peer review Not commissioned; externally peer reviewed.

Open access This is an open access article distributed in accordance with the Creative Commons Attribution Non Commercial (CC BY-NC 4.0) license, which permits others to distribute, remix, adapt, build upon this work non-commercially, and license their derivative works on different terms, provided the original work is properly cited, appropriate credit is given, any changes made indicated, and the use is non-commercial. See: http://creativecommons.org/licenses/by-nc/4.0/.

\section{ORCID iD}

Chad Sloley http://orcid.org/0000-0001-7911-5254

\section{REFERENCES}

1 International Classification of Diseases 11 [ICD-11]. MG30 Chronic Pain, 2019. Available: https://icd.who.int/browse11/l-m/en\#/http://id. who.int/icd/entity/1581976053

2 Healthinfo. Overview of persistent pain, 2018. Available: https://www healthinfo.org.nz/index.htm?Chronic-pain.htm [Accessed cited 2019 5th May].

3 Edwards RR, Dworkin RH, Sullivan MD, et al. The role of psychosocial processes in the development and maintenance of chronic pain. J Pain 2016;17:T70-92.

4 Gatchel RJ. Psychological disorders and chronic pain: cause-andeffect relationships. In: Turk DC, ed. Psychological approaches to pain management: a practitioner's handbook. New York: The Guilford Press, 1996.

5 Fornaro M, Gabrielli F, Albano C, et al. Obsessive-Compulsive disorder and related disorders: a comprehensive survey. Ann Gen Psychiatry 2009;8:13.

6 Fullana MA, Mataix-Cols D, Caspi A, et al. Obsessions and compulsions in the community: prevalence, interference, helpseeking, developmental stability, and co-occurring psychiatric conditions. Am J Psychiatry 2009;166:329-36.

7 Ruscio AM, Stein DJ, Chiu WT, et al. The epidemiology of obsessivecompulsive disorder in the National comorbidity survey replication. Mol Psychiatry 2010;15:53-63.

8 Williams M, Steever A. Cultural manifestations of obsessivecompulsive disorder. In: Lack CW, ed. Obsessive-compulsive disorder: etiology, phenomenology and treatment. Ginger Prince Publications, 2015: 63-84.

9 Guzick AG, Reid AM, Balki AM, et al. Diagnostic description and prevalence. In: Abramowitz JS, McKay D, Storch EA, eds. The wiley handbook of obsessive compulsive disorders. Chichester, UK: John Wiley \& Sons Ltd, 2017: 24-43.

10 American Psychiatric Association. Diagnostic and statistical manual of mental disorders. 5th edn. Arlington, VA: American Psychiatric Association, 2013.

11 International Classification of Diseases 11 [ICD-11]. $6 B 20$ ObsessiveCompulsive Disorder, 2020 11/03/2021]. Available: https://icd.who. int/browse11/l-m/en\#/http\%3a\%2f\%2fid.who.int\%2ficd\%2fentity\% $2 f 1582741816$

12 Mehraban A, Shams J, Moamenzade S, et al. The high prevalence of obsessive-compulsive disorder in patients with chronic pain Iran. $J$ Psychiatry 2014;9:203-8.

13 Calamari JE, Chik HM, Pontarelli NK. Phenomenology and epidemiology of obsessive compulsive disorder. In: Steketee G, ed. The Oxford Handbook of obsessive compulsive and spectrum disorders. New York: Oxford University Press, 2012: 11-47.

14 Reuman L, Jacoby RJ, Blakey SM, et al. Predictors of illness anxiety symptoms in patients with obsessive compulsive disorder. Psychiatry Res 2017;256:417-22.

15 Singh H, Kashyap S, Sivakanthan A. An overview of obsessive compulsive disorder and suicidal behaviour. Open J Psychiatry Allied Sci 2018;9:92-7.

16 Jordan KD, Okifuji A. Anxiety disorders: differential diagnosis and their relationship to chronic pain. J Pain Palliat Care Pharmacother 2011;25:231-45.

17 Huppert JD, Simpson HB, Nissenson KJ, et al. Quality of life and functional impairment in obsessive-compulsive disorder: a comparison of patients with and without comorbidity, patients in remission, and healthy controls. Depress Anxiety 2009;26:39-45.

18 Admon R, Bleich-Cohen M, Weizmant R, et al. Functional and structural neural indices of risk aversion in obsessive-compulsive disorder (OCD). Psychiatry Res 2012;203:207-13.

19 Casado Y, Cobos P, Godoy A, et al. Emotional processing in obsessive-compulsive disorder. J Anxiety Disord 2011;25:1068-71.

20 Watkins ER, Rumination D. Depressive rumination and co-morbidity: evidence for brooding as a transdiagnostic process. J Ration Emot Cogn Behav Ther 2009;27:160-75.

21 Sullivan MJ, Rodgers WM, Kirsch I. Catastrophizing, depression and expectancies for pain and emotional distress. Pain 2001;91:147-54.

22 Calzà J, Gürsel DA, Schmitz-Koep B, et al. Altered cortico-striatal functional connectivity during resting state in obsessive-compulsive disorder. Front Psychiatry 2019;10:319.

23 Fettes P, Schulze L, Downar J. Cortico-striatal-thalamic loop circuits of the orbitofrontal cortex: promising therapeutic targets in psychiatric illness. Front Syst Neurosci 2017;11:25

24 Lipton DM, Gonzales BJ, Citri A. Dorsal striatal circuits for habits, compulsions and addictions. Front Syst Neurosci 2019;13:28

25 Mlost J, Wasik A, Starowicz K. Role of endocannabinoid system in dopamine signalling within the reward circuits affected by chronic pain. Pharmacol Res 2019;143:40-7.

26 Graybiel AM. Habits, rituals, and the evaluative brain. Annu Rev Neurosci 2008;31:359-87.

27 Larson MJ, Clawson A. A critical review of neuropsychological functioning and heterogeneity in individuals with obsessivecompulsive disorder. In: Abramowitz JS, McKay D, Storch EA, eds. The wiley handbook of obsessive compulsive disorders. Chichester UK: John Wiley \& Sons Ltd, 2017: 155-75.

28 Greisberg S, McKay D. Neuropsychology of obsessive-compulsive disorder: a review and treatment implications. Clin Psychol Rev 2003;23:95-117.

29 Tükel R, Gürvit H, Ertekin BA, et al. Neuropsychological function in obsessive-compulsive disorder. Compr Psychiatry 2012;53:167-75.

30 Samuels J, Bienvenu OJ, Krasnow J, et al. An investigation of doubt in obsessive-compulsive disorder. Compr Psychiatry 2017;75:117-24.

31 Ezrati O, Friedman J, Dar R. Attenuation of access to internal states in high obsessive-compulsive individuals might increase susceptibility to false feedback: evidence from a visuo-motor handreaching task. J Behav Ther Exp Psychiatry 2019;65:101445.

32 Lazarov A, Dar R, Liberman N, et al. Obsessive-compulsive tendencies may be associated with attenuated access to internal states: evidence from a biofeedback-aided muscle tensing task. Conscious Cogn 2012;21:1401-9.

33 Lazarov A, Liberman N, Hermesh $\mathrm{H}$, et al. Seeking proxies for internal states in obsessive-compulsive disorder. J Abnorm Psychol 2014;123:695-704

34 Foa EB, Huppert JD, Leiberg S, et al. The obsessive-compulsive inventory: development and validation of a short version. Psychol Assess 2002;14:485-96.

35 Rapp AM, Bergman RL, Piacentini J, et al. Evidence-based assessment of obsessive-compulsive disorder. J Cent Nerv Syst Dis 2016;8:13-29.

36 Hajcak G, Huppert JD, Simons RF, et al. Psychometric properties of the OCI-R in a College sample. Behav Res Ther 2004;42:115-23.

37 Roberts ME, Wilson MS. Factor structure and response bias of the obsessive-compulsive inventory-revised (OCl-R) in a female undergraduate sample from New Zealand. Journal of Psychology 2008;37:2-7. 
38 Obsessive Compulsive Cognitions Working Group. Psychometric validation of the obsessive belief questionnaire and interpretation of intrusions inventory--Part 2: factor analyses and testing of a brief version. Behav Res Ther 2005;43:1527-42.

39 Myers SG, Fisher PL, Wells A. Belief domains of the obsessive beliefs questionnaire-44 (OBQ-44) and their specific relationship with obsessive-compulsive symptoms. J Anxiety Disord 2008;22:475-84

40 Tardif $\mathrm{H}$, Arnold C, Hayes C, et al. Establishment of the Australasian electronic persistent pain outcomes collaboration. Pain Med 2017;18:1007-18.

41 University of Wollongong. Electronic persistent pain outcomes collaboration, 2019. Available: https://ahsri.uow.edu.au/eppoc/index. html

42 Nicholas MK. The pain self-efficacy questionnaire: taking pain into account. Eur J Pain 2007;11:153-63.
43 Sullivan MJL, Bishop SR, Pivik J. The pain catastrophizing scale: development and validation. Psychol Assess 1995;7:524-32.

44 Osman A, Wong JL, Bagge CL, et al. The depression anxiety stress scales-21 (DASS-21): further examination of dimensions, scale reliability, and correlates. J Clin Psychol 2012;68:1322-38.

45 Wood BM, Nicholas MK, Blyth F, et al. The utility of the short version of the depression anxiety stress scales (DASS-21) in elderly patients with persistent pain: does age make a difference? Pain Med 2010;11:1780-90.

46 Hanley JA. Simple and multiple linear regression: sample size considerations. J Clin Epidemiol 2016;79:112-9.

47 Alase A. The interpretative phenomenological analysis (IPA): a guide to a good qualitative research approach. IJELS 2017;5:9-19.

48 Smith JA, Osborn M. Interpretative phenomenological analysis. In: Smith JA, ed. Qualitative psychology: a practical guide to research methods. Los Angeles: SAGE, 2015: 25-52. 